Pacific Journal of Mathematics

A CRITERION FOR DIVISORIALITY 


\section{A CRITERION FOR DIVISORIALITY}

\section{Michael ENGBer}

In this paper a criterion for divisoriality of a scheme is given. The corresponding relative notion is defined and a criterion is given in this case as well.

1. In [2], Borelli proves that any coherent sheaf on a divisorial scheme is the quotient of a locally free sheaf. An examination of his proof reveals that he actually proves the stronger result that any quasi-coherent sheaf of finite type on a divisorial scheme is the quotient of a direct sum of invertible sheaves. Furthermore, these invertible sheaves are tensor powers of the inverses of the sheaves giving the scheme as divisorial.

The purpose of this note is to prove the (weaker) converse of this stronger result. In $\S 3$, we define the notion of divisorial morphism and prove an analogous characterization theorem. The author would like to express his gratitude to Steven Kleiman who made several helpful suggestions.

Recall that $X$ is divisorial if and only if, for every $x \in X$ there exists an invertible sheaf $\mathscr{L}$ on $X$, an element $s \in \Gamma\left(X, \mathscr{L}^{\otimes m}\right)$ such that $X_{s}$ is affine and $x \in X_{s}$. If $\mathscr{L}$ is any invertible sheaf on $X$, we write

$$
X_{\mathscr{L}}=\bigcup\left\{X_{s} \mid X_{s} \text { affine, } s \in \Gamma\left(X, \mathscr{L}^{\otimes m}\right), m=1,2,3, \cdots\right\} \text {. }
$$

With this notation, $X$ is divisorial if and only if there exists a finite number of invertible sheaves $\mathscr{L}_{1}, \cdots, \mathscr{L}_{n}$ such that $X=\mathrm{U} \mathscr{L}_{i}$. In this case, we will say that $X$ is divisorial for $\mathscr{L}_{1}, \cdots, \mathscr{L}_{n}$.

2. Theorem 1. Let $X$ be a scheme and let $\mathscr{L}_{1}, \cdots, \mathscr{L}_{n}$ be invertible sheaves on $X$. Suppose that for any quasi-coherent sheaf $\mathscr{F}$ of finite type, there exists a surjection $\mathscr{E} \rightarrow \mathscr{F}$ where $\mathscr{E}$ is a direct sum of tensor powers of the inverses of the $\mathscr{L}_{i}$. Then $X$ is divisorial for $\mathscr{L}_{1}, \cdots, \mathscr{L}_{n}$.

Proof. By Corollary 3.1 of [1], it suffices to show that the family $\left\{X_{s} \mid s \in \Gamma\left(X, \mathscr{L}_{i}^{\otimes m}\right) ; i=1, \cdots, n ; m=1,2,3, \cdots\right\}$ forms a base for the topology of $X$. To see this, let $U$ be an open subset of $X$ and let $x \in U$. If $Y=X-U$ and $\mathscr{F}$ is the Ideal of $\mathcal{O}$ corresponding to $Y$, then $\mathscr{J}$ is quasi-coherent. Since $X$ is a scheme, $\mathscr{J}$ is the direct limit of its quasi-coherent sub-Ideals $\mathscr{F}_{2}$ of finite type ([3] I, 9.4.9). Each $\mathscr{F}_{\lambda}$ corresponds to a closed subscheme $Y_{\lambda} \supset Y$. If $x$ were contained in each $Y_{\lambda}$, then $x$ would be an element of $Y$. But $x \in U$ so 
there exists some $Y_{\lambda} \supset Y$ such that $x \notin Y_{\lambda}$ and the associated Ideal is of finite type. Thus, we may assume that $I$ itself is of finite type.

By hypothesis, there exists a surjection $\mathscr{E} \rightarrow \mathscr{J}$ where $\mathscr{E}$ is a direct sum of tensor powers of the inverses of the $\mathscr{L}_{i}$. For convenience, let us write $\mathscr{E}$ as $\mathscr{L}_{1}^{\prime} \oplus \cdots \oplus \mathscr{L}_{t}^{\prime}$. The surjection $\mathscr{E} \rightarrow \mathscr{J}$ induces a surjection $\gamma: \mathscr{E}_{x} \rightarrow \mathscr{I}_{x}$ of stalks at $x$ and maps $\gamma_{j}: \mathscr{L}_{j, x}^{\prime} \rightarrow$ $\mathscr{F}_{x}=\mathscr{O}_{x}$ (since $x \notin Y$ ). We claim that at least one of the $\gamma_{j}$ is surjective since, if not, the images of all the $\gamma_{j}$ would be contained in the maximal ideal of $\mathscr{O}_{x}$ and this would imply that the image of $\gamma$ itself is contained in the maximal ideal. Let $\alpha$ denote the composite map of sheaves $\mathscr{L}_{j}^{\prime} \rightarrow \mathscr{J} \rightarrow \mathscr{O}$ where the second map is the canonical inclusion and $j$ is chosen so that $\gamma_{j}$ is surjective. Taking the tensor product of $\alpha$ with $\mathscr{L}_{j}^{\prime-1}$, we obtain $\alpha^{\prime}: \mathcal{O} \rightarrow \mathscr{L}_{j}^{\prime-1}$ which is therefore defined by some $s \in \Gamma\left(X, \mathscr{L}_{j}^{\prime-1}\right)$. Since $\alpha^{\prime}$ is surjective on the stalk at $x$, it follows that $s(x) \neq 0$. On the other hand, for all $y \in Y$, the image of $\alpha_{y}$ is contained in $\mathscr{J}_{y}$ and hence in the maximal ideal of $\mathcal{O}_{y}$, so that $s(y)=0$. Thus $x \in X_{s} \subset U$. Realizing that $\mathscr{L}_{j}^{\prime-1}$ is a tensor power of one of the $\mathscr{L}_{i}$ completes the proof.

3. The notion of divisoriality has a relativization entirely analogous to the notion of relative ampleness given in [3] II, 4.6.1.

Definition: Let $f: X \rightarrow Y$ be a morphism of schemes and let $\mathscr{L}_{1}, \cdots, \mathscr{L}_{n}$ be invertible sheaves on $X$. We say that $f$ is divisorial for $\mathscr{L}_{1}, \cdots, \mathscr{L}_{n}$ or that $X$ is divisorial over $Y$ for $\mathscr{L}_{1}, \cdots, \mathscr{L}_{n}$ provided that there exists an open affine cover of $Y$ such that $V=f^{-1}(U)$ is divisorial for $\mathscr{L}_{1}\left|V, \cdots, \mathscr{L}_{n}\right| V$ for every $U$ in the cover.

Note that every such $f$ will automatically be separated and quasicompact.

THEOREM 2. Let $f: X \rightarrow Y$ be a separated, quasi-compact morphism of schemes and let $\mathscr{L}_{1}, \cdots, \mathscr{L}_{n}$ be invertible sheaves on $X$. Then $f$ is divisorial for $\mathscr{L}_{1}, \cdots, \mathscr{L}_{n}$ if and only if, for every quasicoherent $\mathcal{O}_{X}$-Module $\mathscr{F}$ of finite type, there exists a surjection $\mathscr{E} \rightarrow$ $\mathscr{F}$ where $\mathscr{E}$ is a direct sum of sheaves of the form $\mathscr{L}_{j}^{\otimes-m} \otimes f^{*}(\mathscr{G})$ and each $\mathscr{G}$ is a quasi-coherent $\mathcal{O}_{Y}$-Module of finite type.

Proof. Suppose $f$ is divisorial. Let $U$ be an element of the open affine cover of $Y$ which exhibits the divisoriality of $f$ and let $V=$ $f^{-1}(U)$. By definition, $V$ can be covered by finitely many sets of the form $V_{\overline{\mathscr{L}}}$ where $\overline{\mathscr{L}}=\mathscr{L} \mid V$. By Lemma 3.2 of [2], for a sufficiently large integer $m, \mathscr{F} \otimes \mathscr{L}^{\otimes m} \mid V_{\overline{\mathscr{S}}}$ is generated by finitely many elements of $\Gamma\left(V, \mathscr{F} \otimes \mathscr{L}^{\otimes m}\right)$. But then, the canonical morphism $(f \mid V)^{*}(f \mid V)_{*}\left(\mathscr{F} \otimes \mathscr{L}^{\otimes m} \mid V\right) \rightarrow \mathscr{F} \otimes \mathscr{L}^{\otimes m} \mid V$ is surjective on $V_{\overline{\mathscr{P}}}$ 
by [3] $0_{I}$, 5.1.2. Now the first member is just $f^{*} f_{*}\left(\mathscr{F} \otimes \mathscr{L}^{\otimes m}\right) \mid V$ so that we can say that the global morphism $f^{*} f_{*}\left(\mathscr{F} \otimes \mathscr{L}^{\otimes m}\right) \rightarrow$ $\mathscr{F} \otimes \mathscr{L}^{\otimes m}$ is surjective on $V_{\overline{\mathscr{C}}}$. Since $f$ is quasi-coherent and separated, $f_{*}\left(\mathscr{F} \otimes \mathscr{L}^{\otimes m}\right)$ is quasi-coherent on $Y([3] \mathrm{I}, 9.2 .2)$ whence it is the direct limit of its quasi-coherent sub- $\mathscr{O}_{Y}$-Modules $\mathscr{G}_{\lambda}$ of finite type ([3] I, 9.4.9). Since inverse image commutes with direct limit, we have $f^{*} f_{*}\left(\mathscr{F} \otimes \mathscr{L}^{\otimes m}\right)=\operatorname{dir}$. lim. $f^{*}\left(\mathscr{G}_{\lambda}\right)$. Since $X$ is quasi-compact, $\mathscr{F} \otimes \mathscr{L}^{\otimes m}$ of finite type and the map $f^{*} f_{*}\left(\mathscr{F} \otimes \mathscr{L}^{\otimes m}\right) \rightarrow \mathscr{F} \otimes \mathscr{L}^{\otimes m}$ surjective on $V_{\overline{\mathscr{S}}}$ it follows that there exists an index $\lambda$ such that $f^{*}\left(\mathscr{G}_{\lambda}\right) \rightarrow \mathscr{F} \otimes \mathscr{L}^{\otimes m}$ is surjective on $V_{\overline{\mathscr{L}}}\left([3] 0_{I}, 5.2 .3\right)$. But then $\mathscr{L}^{\otimes-m} \otimes f^{*}\left(\mathscr{G}_{\lambda}\right) \rightarrow \mathscr{F}$ is surjective on $V_{\overline{\mathscr{L}}}$ and since $X$ is covered by finitely many sets of the form $V_{\overline{\mathscr{L}}}$, the result follows.

To prove sufficiency, let $U$ be an arbitrary open affine subset of $Y$ and let $V=f^{-1}(U)$. We will show that $V$ is divisorial for $\mathscr{L}_{1}\left|V, \cdots, \mathscr{L}_{n}\right| V$ by the criterion of Theorem 1 . To this end, let $\mathscr{F}$ be any quasi-coherent Module of finite type on $V$. By [3] I, 9.4.8, there exists a quasi-coherent Module $\mathscr{F}^{\prime}$ of finite type on $X$ such that $\mathscr{F}^{\prime} \mid V=\mathscr{F}$.

By hypothesis, there exists a surjection

$$
\mathscr{L}_{1}^{\prime} \otimes f^{*}\left(\mathscr{G}_{1}\right) \oplus \cdots \oplus \mathscr{L}_{t}^{\prime} \otimes f^{*}\left(\mathscr{G}_{t}\right) \longrightarrow \mathscr{F}^{\prime}
$$

where we have written $\mathscr{L}_{j}^{\prime}$ to represent negative tensor powers of the $\mathscr{L}_{i}$ as we did in the proof of Theorem 1. The above map is, of course, still a surjection when restricted to $V$ and we will write $\overline{\mathscr{L}^{\prime}}=\mathscr{L}^{\prime} \mid V$. Since the $\mathscr{G}_{j}$ are quasi-coherent and $U$ is affine, $\mathscr{G}_{j} \mid U$ is generated by its global sections whence we have surjections $\mathscr{O}_{U}^{r_{j}} \rightarrow$ $\mathscr{G}_{j} \mid U$ and, taking inverse images by $f \mid V, \mathcal{O}_{V}^{r_{j}} \rightarrow(f \mid V)^{*}\left(\mathscr{G}_{j} \mid U\right)=$ $f^{*}\left(\mathscr{G}_{j}\right) \mid V$. In this way, we obtain surjections

$$
\left(\overline{\mathscr{L}}_{j}^{\prime}\right)^{r_{j}}=\overline{\mathscr{L}}_{j}^{\prime} \otimes{\mathcal{O}^{r}}^{r_{j}} \longrightarrow \overline{\mathscr{L}}_{j}^{\prime} \otimes f^{*}\left(\mathscr{G}_{j}\right)\left|V=\left(\mathscr{L}_{j}^{\prime} \otimes f^{*}\left(\mathscr{G}_{j}\right)\right)\right| V .
$$

Finally, by taking direct sums over $j$ and composing with the restriction of the given surjection to $V$, we obtain

$$
\left(\overline{\mathscr{L}}_{1}^{\prime}\right)^{r_{1}} \oplus \cdots \oplus\left(\overline{\mathscr{L}}_{t}^{\prime}\right)^{r_{t}} \longrightarrow \mathscr{F} \longrightarrow 0 \text { is exact on } V \text {. }
$$

Since $\mathscr{F}$ was arbitrary, this implies that $V$ is divisorial for $\overline{\mathscr{L}}_{1}, \cdots, \overline{\mathscr{L}}_{n}$. Since $U$ can be taken as an element of an affine cover of $Y$, the result follows.

Note. The above results remain valid if the hypothesis of separation is relaxed to quasi-separation in view of the results of [3] IV, 1.7. 


\section{REFERENCES}

1. M. Borelli, Divisorial varieties, Pacific J. Math., 13 (1963), 375-388.

2. - Some results on ampleness and divisorial schemes, Pacific J. Math., 23 (1967), 217-227.

3. A. Grothendieck and J. Dieudonné, Eléments de Géométrie Algébrique, Publ. Math. de l'I.H.E.S. 4, 8, 20, Paris, 1960, 1964.

Received December 13, 1971.

City University of New York 


\section{PACIFIC JOURNAL OF MATHEMATICS}

EDITORS

\author{
H. SAMELSON \\ Stanford University \\ Stanford, California 94305 \\ C. R. HOBBY \\ University of Washington \\ Seattle, Washington 98105
}

\author{
J. DUGUNDJI \\ Department of Mathematics \\ University of Southern California \\ Los Angeles, California 90007 \\ RICHARD ARENS \\ University of California \\ Los Angeles, California 90024
}

\section{ASSOCIATE EDITORS}

E. F. BECKENBACH

B. H. NeUManN

F. WOLF

K. YosHIDA

\section{SUPPORTING INSTITUTIONS}

UNIVERSITY OF BRITISH COLUMBIA

CALIFORNIA INSTITUTE OF TECHNOLOGY

UNIVERSITY OF CALIFORNIA

MONTANA STATE UNIVERSITY

UNIVERSITY OF NEVADA

NEW MEXICO STATE UNIVERSITY

OREGON STATE UNIVERSITY

UNIVERSITY OF OREGON

OSAKA UNIVERSITY
UNIVERSITY OF SOUTHERN CALIFORNIA

STANFORD UNIVERSITY

UNIVERSITY OF TOKYO

UNIVERSITY OF UTAH

WASHINGTON STATE UNIVERSITY UNIVERSITY OF WASHINGTON

AMERICAN MATHEMATICAL SOCIETY NAVAL WEAPONS CENTER 


\section{Pacific Journal of Mathematics}

\section{Vol. 45, No. $1 \quad$ September, 1973}

William George Bade, Complementation problems for the Baire classes .......... 1

Ian Douglas Brown, Representation of finitely generated nilpotent groups ........ 13

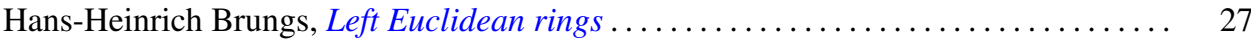

Victor P. Camillo and John Cozzens, A theorem on Noetherian hereditary rings ..... 35

James Cecil Cantrell, Codimension one embeddings of manifolds with locally flat

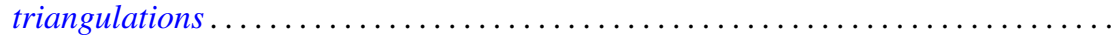

L. Carlitz, Enumeration of up-down permutations by number of rises . . . . . . . . . .

Thomas Ashland Chapman, Surgery and handle straightening in Hilbert cube

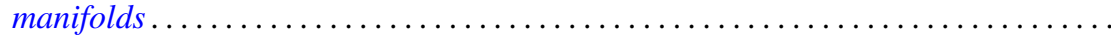

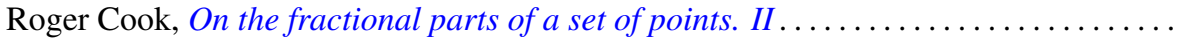

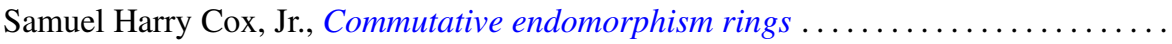

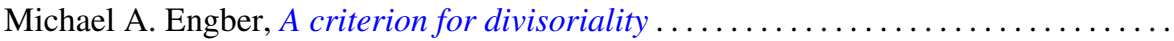

Carl Clifton Faith, When are proper cyclics injective . . . . . . . . . . . . . . 97

David Finkel, Local control and factorization of the focal subgroup . . . . . . . . . 113

Theodore William Gamelin and John Brady Garnett, Bounded approximation by

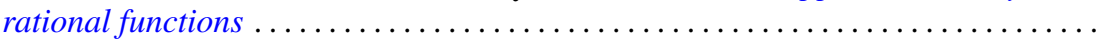

Kazimierz Goebel, On the minimal displacement of points under Lipschitzian

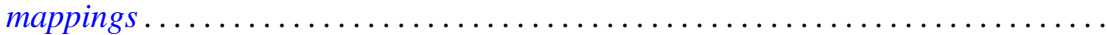

Frederick Paul Greenleaf and Martin Allen Moskowitz, Cyclic vectors for representations associated with positive definite measures: nonseparable

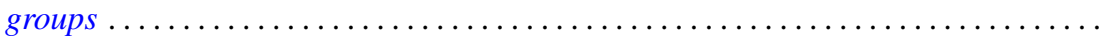

Thomas Guy Hallam and Nelson Onuchic, Asymptotic relations between perturbed linear systems of ordinary differential equations .

David Kent Harrison and Hoyt D. Warner, Infinite primes of fields and completions. .

James Michael Hornell, Divisorial complete intersections . ......

Jan W. Jaworowski, Equivariant extensions of maps ..............

John Jobe, Dendrites, dimension, and the inverse arc function .. .

Gerald William Johnson and David Lee Skoug, Feynman integrals of non-factorable

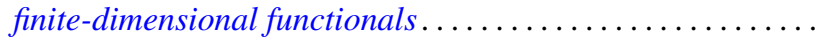

Dong S. Kim, A boundary for the algebras of bounded holomorphic functions ...... 269

Abel Klein, Renormalized products of the generalized free field and its derivatives ... 275

Joseph Michael Lambert, Simultaneous approximation and interpolation in $L_{1}$ and

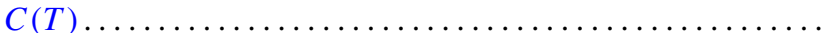

Kelly Denis McKennon, Multipliers of type $(p, p)$ and multipliers of the group

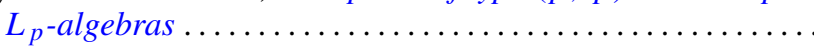

William Charles Nemitz and Thomas Paul Whaley, Varieties of implicative

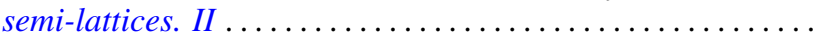

Donald Steven Passman, Some isolated subsets of infinite solvable

Norma Mary Piacun and Li Pi Su, Wallman compactifications on E-completely

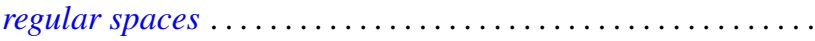

Jack Ray Porter and Charles I. Votaw, $S(\alpha)$ spaces and regular Hausdorff extensions....

Gary Sampson, Two-sided $L_{p}$ estimates of convolution transforms .

Ralph Edwin Showalter, Equations with operators forming a rig
Raymond Earl Smithson, Fixed points in partially ordered sets .

Victor Snaith and John James Ucci, Three remarks on symmetric products and

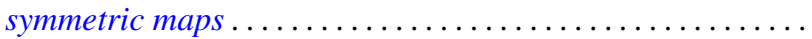

